\title{
Description of Treatment of Uterus Cancer Based on the Japanese DPC Database
}

\author{
Keiji Muramatsu' ${ }^{1)}$, Shinya Matsuda ${ }^{1,2)}$, Kenshi Hayashida ${ }^{2)}$, Tatsuhiko Kubo'), \\ Yoshihisa Fujino ${ }^{1)}$, Kenji Fujimori ${ }^{3)}$, Kiyohide Fushimi ${ }^{4)}$ \\ ${ }^{1)}$ Department of Preventive Medicine and Community Health, School of Medicine, University of Occupational \\ and Environmental Health \\ ${ }^{2)}$ Medical Informatics Division, University Hospital of University of Occupational and Environmental Health \\ ${ }^{3)}$ Center for Regional Healthcare and Certified Educator Support, Hokkaido University \\ ${ }^{4)}$ Health Policy and Informatics Section, Department of Health Policy, Tokyo Medical and Dental University
}

\begin{abstract}
BACKGROUND: Cancer has been the leading cause of death in Japan since 1981 and the number of cancer deaths in 2010 in Japan was about 354,000. In 2002, The Japanese government has introduced the casemix based evaluation system. This made it possible to use the casemix database for the evaluation of clinical process of cancer treatment. We tried to describe the treatment process of uterus cancer among the Japanese acute care hospitals. MATERIAL AND METHOD: We have extracted 36,490 uterus cancer cases from the DPC database (1st July 12010 to 31st October 2010). Using this dataset, we have described the cases according to age category, cancer stage and contents of treatment. RESULTS: Among all cases, cervical cancer cases were 14,498, corpus cancer cases were 15,322 and other types (including unknown) were 6,670 . Both corpus and cervical cancer, stage 1 is the most frequent for primary cases. For recurrent cases of corpus cancer, stage 3 was the most frequent (34\%) and for cervical cancer, stage 2 was the most frequent (29\%). In both case of corpus and cervical cancer, the most frequent regimen was "carboplatin + paclitaxel". CONCLUSION: By using DPC data, it is possible to describe the treatment process of uterus cancer among the Japanese acute care hospitals. If it become to be possible to combine DPC database with other large scale clinical data, it will be an important information resource to complement such clinical database.
\end{abstract}

Key words: DPC, casemix, uterus cancer, EBM, Japan

\section{*ntroduction}

Cancer has been the leading cause of death in Japan since 1981. The number of cancer deaths in 2010 in Japan was about 354,000. In terms of cancer sites, lung was the leading site (23.8\%) for males, followed by stomach (15.6\%), liver (10.2\%), colon (7.1\% ; same rank when colon and rectum are com-

Received: May 20, 2012

Accepted: June 5, 2013

Correspondence: K. Muramatsu, 1-1 Iseigaoka, Yahatanishiku, Kitakyushu, 807-8555, Fukuoka, Japan

e-mail: km@med.uoeh-u.ac.jp bined : $11.4 \%)$, and pancreas (6.9\%). The leading site for females was lung $(13.7 \%)$, followed by stomach $(12.1 \%)$, colon $(10.6 \%$; same rank when colon and rectum are combined : $14.4 \%)$, pancreas $(9.5 \%)$, and breast $(8.8 \%)^{1)}$.

Considering the importance of dealing with cancer for the population's health, the Japanese government has been implemented the 3 rd-term Comprehensive 10-year Strategy for Cancer Control since 2004. The main purpose is to largely reduce the morbidity and mortality of cancer by promoting comprehensive health care, prevention and research.

In June 2006, the Cancer Control Act was 
approved and the law has been implemented since April 2007. The basic concepts of the law are 1) promotion of cancer research and utilization of research outcomes, 2) equalization of cancer medical services, and 3) development of cancer medical services to satisfy patients. Furthermore, according to this law, the Japanese government constructed the Basic Plan in order to promote Cancer Control Programs in June 2007, covering five fiscal years from 2007 to 2011. The plan defines the basic concept of cancer control and aims to promote comprehensive and well-planned cancer control in Japan.

As cancer is the leading cause of death in Japan and the most important issue of medical research, patients and their family, as well as the general population demand more objective and practical information about cancer treatment. The Japanese government has introduced the casemix based evaluation system in 2002. In 2011, 1650 acute care hospitals submit their casemix related data to the Ministry of Health, Labour and Welfare (MHLW). As this dataset contains very detailed process data for each inpatient case, it is possible to use the casemix database for the evaluation of clinical process of cancer treatment.

For this purpose, the authors tried to describe the treatment process of uterus cancer among the Japanese acute care hospitals.

\section{Materials and Method}

Data for this study were extracted from the Japanese inpatient administrative claims database, the DPC database ${ }^{2}$. The database was originally instituted as part of a national project to develop a Japanese case-mix classification system, which has been ongoing since 2002. The annual number of cases in the database is approximately three million. The database contains: i) main diagnoses, pre-existing comorbidities at admission and complications after admission which are coded with ICD-10 codes; ii) surgical procedures coded with Japanese original codes (Kcodes), operation time and the performed date; iii) discharge status (dead or alive); and iv) a list of drugs and blood products used and the dates of use. Study approval was obtained from the Institutional Review Boards and the Ethics Committee of The Tokyo Medical and Dental University. Given the anonymous nature of the data collection process, informed con- sent was not required.

For this study, we have extracted 36,490 uterus cancer cases from the DPC database (1st July 12010 to $31 \mathrm{~s}^{\mathrm{t}}$ October 2010). Using this dataset, we have described the cases according to age category, cancer stage and contents of treatment. Statistical analyses were conducted using IBM SPSS version 19.0 (IBM SPSS, Armonk, NY, USA).

\section{Results}

Among the 36,490 cases, cervical cancer cases were 14,498, corpus cancer cases were 15,322 and other types (including unknown) were 6,670. Average ages were 51.2 (SD: 14.9), 60.3 (SD: 11.5) and 39.7 (SD: 11.2), respectively.

Table 1 shows the number of cases according to primary/recurrent, stage, and type of cancers. For primary cases of corpus cancer, stage 1 is the most frequent (40\%) followed by stage 3 (24\%), unknown $(16 \%)$, and stage $4(12 \%)$. For primary case of cervical cancer, stage 1 is the most frequent followed by stage $0(20 \%)$, stage $2(16 \%)$, unknown (16\%) and stage 3 (13\%). For recurrent cases of corpus cancer, stage 3 was the most frequent (34\%) followed by stage 4 $(25 \%)$ and stage $1(21 \%)$. For cervical cancer, stage 2 was the most frequent (29\%) followed by stage 3 $(21 \%)$ and stage $1(21 \%)$ and stage $4(19 \%)$. Corpus cancer cases tend to be in more advanced stages.

Among the cases, the most frequent DPC was 120020xx99x40x "Uterus cancer, no surgical procedure, with chemotherapy, without radiotherapy, without CC" $(7,496$ cases, $30.4 \%)$, followed by 120020xx02x0xx "Uterus cancer, partial resection, without additional procedure, without chemotherapy, without radiotherapy" (6,095 cases, $24.7 \%)$ and 120020xx02x0xx "Uterus cancer, cancer surgery, without additional procedure, without chemotherapy, without radiotherapy" (3,605 cases, 14.6\%).

Concerning to choice of surgical procedures both for corpus and cervical cancers, for primary corpus cancer cases, $55.5 \%$ (6302 of 11,360 cases) were nonsurgical and $31.7 \%$ (3,603 cases) received total hysterectomy. In the case of cervical cancer, $43.1 \%$ $(4,646$ of 10,775 cases $)$ were non-surgical, $26.1 \%$ (2,515 cases) received partial hysterectomy and $22.7 \%$ (2,441 cases) received total hysterectomy. For recurrent corpus cancer cases, $82.8 \%$ (3,241 of 3,914 cases) were non-surgical and $12.0 \%$ (471 cases) 
Table 1 Number of cases according to primary/recurrent, stage, and type of cancers (1st July 12010 to 31 st October 2010)

\begin{tabular}{|c|c|c|c|c|c|c|c|c|}
\hline & & Stage0 & Stage1 & Stage2 & Stage3 & Stage4 & Unknown & Total \\
\hline \multirow[t]{8}{*}{ Primary } & \multirow[t]{2}{*}{ Others } & 45 & 10 & 2 & 12 & 8 & 62 & 139 \\
\hline & & $32 \%$ & $7 \%$ & $1 \%$ & $9 \%$ & $6 \%$ & $45 \%$ & $100 \%$ \\
\hline & \multirow[t]{2}{*}{ Corpus } & 81 & 1,824 & 294 & 1,085 & 550 & 750 & 4,584 \\
\hline & & $2 \%$ & $40 \%$ & $6 \%$ & $24 \%$ & $12 \%$ & $16 \%$ & $100 \%$ \\
\hline & \multirow[t]{2}{*}{ Cervical } & 807 & 965 & 670 & 518 & 487 & 658 & 4,105 \\
\hline & & $20 \%$ & $24 \%$ & $16 \%$ & $13 \%$ & $12 \%$ & $16 \%$ & $100 \%$ \\
\hline & \multirow[t]{2}{*}{ Total } & 933 & 2,799 & 966 & 1,615 & 1,045 & 1,470 & 8,828 \\
\hline & & $11 \%$ & $32 \%$ & $11 \%$ & $18 \%$ & $12 \%$ & $17 \%$ & $100 \%$ \\
\hline \multirow[t]{8}{*}{ Recurrent } & \multirow[t]{2}{*}{ Others } & 1 & 1 & 3 & 2 & 7 & 6 & 20 \\
\hline & & $5 \%$ & $5 \%$ & $15 \%$ & $10 \%$ & $35 \%$ & $30 \%$ & $100 \%$ \\
\hline & \multirow[t]{2}{*}{ Corpus } & 3 & 124 & 43 & 206 & 152 & 75 & 603 \\
\hline & & $0 \%$ & $21 \%$ & $7 \%$ & $34 \%$ & $25 \%$ & $12 \%$ & $100 \%$ \\
\hline & \multirow[t]{2}{*}{ Cervical } & 7 & 151 & 208 & 154 & 141 & 65 & 726 \\
\hline & & $1 \%$ & $21 \%$ & $29 \%$ & $21 \%$ & $19 \%$ & $9 \%$ & $100 \%$ \\
\hline & \multirow[t]{2}{*}{ Total } & 11 & 276 & 254 & 362 & 300 & 146 & 1,349 \\
\hline & & $1 \%$ & $20 \%$ & $19 \%$ & $27 \%$ & $22 \%$ & $11 \%$ & $100 \%$ \\
\hline
\end{tabular}

received other surgical procedures. For cervical cancer, $75.5 \%$ (2,909 of 3,692 cases) were non-surgical and $16.6 \%$ (614 cases) received other surgical procedures. According to the detailed process analysis, the most frequent "other" procedure was "placement of central venous access port" both for corpus and cervical cancer.

Table 2 showed the regimen of chemotherapy stratified by corpus and cervical cancer. In the case of corpus cancer, the most frequent regimen was "carboplatin + paclitaxel" $(4,481$ of 9,717 cases; $46 \%)$ followed by "doxorubicine+ cisplatine" (690 cases, $7 \%$ ), "carboplatin + paclitaxel + dexamethasone" (642 cases, 7\%), "carboplatin + docetaxel" (642 cases, 7\%) and "carboplatin + docetaxel + dexamethasone" (642 cases, 7\%). For cervical cancer, the most used regimen was "carboplatin + paclitaxel" $(1,536$ of 7,142 cases, 22\%), followed by "cisplatine" $(809,11 \%)$, "irinotecan + nedaplatin" (538, 8\%), "paclitaxel+ cisplatine" (423, 6\%) and irinotecan hydrochloride (413, $6 \%)$.

\section{Discussion}

Cancer has been the leading cause of deaths in Japan, with one person out of three dying of the disease. More people are dying of cancer as the population gets older. The countermeasure for cancer control is an increasingly serious issue for the health policy maker in Japan. However, it is criticized that there are wide variation of cancer care in accessibility and quality among the different regions and institutions.

As Table 2 shows, the most frequent regimen for corpus cancer was "carboplatin + paclitaxel" in Japan. Compared with cisplatine, carboplatin is considered less possibility of causing side effects such as nausea and vomiting. This may explain the current result. That is, clinicians prefer to use carboplatin in order to avoid side effects. Although we cannot clarify which regimen is clinically most effective by the DPC database only, it is very important to recognize that the process of cancer treatment at the DPC hospital can be clarified by using the DPC data.

In order to further ameliorate cancer control policy, MHLW has established "The third term Comprehensive 10-Year Strategy for Cancer Control" in 2006. One of the most important objectives of the Strategy is to assure the equal access to quality cancer treatment for all population. This requires the objective information for citizens about the actual situation of cancer treatment. The DPC framework is can be used for this purpose. Using the summarized results such as Table 2, both clinicians and patients can know what are the main streams of today's cancer treatment, for example. This kind of information will facilitate EBM and Quality Assurance movement in 
Table 2 Number of cases stratified by chemotherapy regimen and cancer type (1st July 12010 to 31 st October 2010)

Cervical cancer: Individual drug

\begin{tabular}{cccccc}
\hline Total & paclitaxel & cisplatine & carboplatin & irinotecan hydrochloride & dexamethasone \\
\hline \multirow{2}{*}{7,142} & 2,729 & 2,683 & 2,190 & 1,601 & 1,350 \\
& $38 \%$ & $38 \%$ & $31 \%$ & $22 \%$ & $19 \%$ \\
\hline
\end{tabular}

Corpus cancer: Individual drug

\begin{tabular}{cccccc}
\hline Total & carboplatin & paclitaxel & cisplatine & dexamethasone & docetaxelhydrate \\
\hline 9,717 & 6,380 & 5,892 & 1,848 & 1,626 & 1,485 \\
& $66 \%$ & $61 \%$ & $19 \%$ & $17 \%$ & $15 \%$ \\
\hline
\end{tabular}

Cervical cancer: regimen

\begin{tabular}{|c|c|c|c|c|c|}
\hline Total & $\begin{array}{l}\text { carboplatin } \\
\text { +paclitaxel }\end{array}$ & cisplatine & $\begin{array}{l}\text { irinotecan } \\
\text { +nedaplatin }\end{array}$ & $\begin{array}{l}\text { paclitaxel } \\
+ \text { cisplatine }\end{array}$ & $\begin{array}{c}\text { irinotecan } \\
\text { hydrochloride }\end{array}$ \\
\hline 7,142 & 1,536 & 809 & 538 & 423 & 413 \\
\hline$\%$ & $22 \%$ & $11 \%$ & $8 \%$ & $6 \%$ & $6 \%$ \\
\hline Cumulative \% & $22 \%$ & $33 \%$ & $40 \%$ & $46 \%$ & $52 \%$ \\
\hline \multicolumn{6}{|c|}{ Corpus cancer: regimen } \\
\hline Total & $\begin{array}{l}\text { carboplatin } \\
+ \text { paclitaxel }\end{array}$ & $\begin{array}{l}\text { doxorubicine } \\
+ \text { cisplatine }\end{array}$ & $\begin{array}{c}\text { carboplatin } \\
\text { +paclitaxel } \\
\text { +dexamethasone }\end{array}$ & $\begin{array}{l}\text { carboplatin } \\
+ \text { docetaxel }\end{array}$ & $\begin{array}{c}\text { carboplatin } \\
+ \text { docetaxel } \\
+ \text { dexamethasone }\end{array}$ \\
\hline 9,717 & 4,481 & 690 & 642 & 575 & 283 \\
\hline$\%$ & $46 \%$ & $7 \%$ & $7 \%$ & $6 \%$ & $3 \%$ \\
\hline Cumulative \% & $46 \%$ & $53 \%$ & $60 \%$ & $66 \%$ & $69 \%$ \\
\hline
\end{tabular}

cancer care.

Several limitations must be considered when interpreting our results. First, we lacked precise information about cancer staging. As the DPC database is an administrative data, clinicians are not enough motivated to register all required data that are not necessary for reimbursement. Furthermore, the DPC data does not have information that becomes available after discharge. This sometimes happens for time-lag for making pathological diagnosis. Second, as the DPC database covers only in-patient, information of chemotherapies at out-patient services is not covered. This might cause a bias. In order to ameliorate the usability of DPC database, MHLW decided to gather the administrative data of out-patient services of DPC hospitals from 2012. This will improve the comprehensiveness of DPC database for clinical researches. Despite these limitations, we believe that DPC data- base has an important potential to advance a large scale clinical studies in Japan. Of course, as DPC data is under the administrative scheme, it is not appropriate to include clinical data that are not directly associated with financial evaluation. However, if we can combine DPC database with other large scale clinical data, it will be an important information resource to complement such clinical database and to reduce the workload of registry. For this purpose, we have tried to combine the DPC database and Stroke registry ${ }^{3}$. This trial has successfully showed the possibility to use the DPC database as a complement resource of Stroke registry system. It is expected that this trial would be adapted to other clinical database.

\section{References}

1. National Cancer Center. http://ganjoho.jp/public/ 
statistics/pub/statistics01.html (Access, 9 June 2012)

2. Matsuda S: Diagnosis Procedure Combination - the Japanese original casemix system-, In: Kimberly J and Pouvourville $\mathrm{G}$ eds, the Globalization of Managerial Innovation in Health Care, Cambridge Uni- versity Press, 2009.

3. Fujimori K, Matsuda S, Horiguchi H: Linkage of DPC database and Stroke registry in Japan, In: Kobayashi S: Report on Study on linkage of emergency transfer resister and clinical record based on the AMI database and Stroke database, 2012. 